\title{
Étude des convertisseurs multicellulaires parallèles : II. Analyse du modèle
}

\author{
Pascal Davancens et Thierry Meynard (*) \\ Laboratoire d'Électrotechnıque et d'Électronıque Industrıelle $\left(^{* *}\right)$, \\ I.N.P. Toulouse - E.N.S.E.E.I.H.T., 2 rue Charles Camichel, 31071 Toulouse Cedex, France
}

(Reçu le 13 mas 1996, révisé le 25 septembre 1996, accepté le 8 octobre 1996)

PACS.02.60.Cb - Numerical simulation

PACS.84.30 Jc - Power electronıcs; power supply circuits

PACS.84.70 +p - High-current and high-voltage technology· power systems

Résumé. - Cet artıcle est basé sur l'étude d'un modèle développé afin de prédıre le comportement des convertısseurs multicellulaires parallèles Ce modèle étant développé dans l'espace d'état, l'étude de la matrice dynamique et plus particulièrement de ses valeurs propres nous permet de caractériser les dynamıques de rééquilibrage du convertisseur. L'obtention d'une relation analytique directe entre les paramètres du convertisseur et les valeurs propres du modèle nous permet de mieux appréhender les mécanismes de rééquilıbrage et de définir des règles de dimensionnement. Ce modèle dans l'espace d'état facilite également l'étude du régime permanent obtenu sans simulation Ains1, nous pouvons montrer la sensibilité du convertisseur aux imperfections de la commande et des valeurs des paramètres de la structure.

\begin{abstract}
This paper deals with the study of the harmonic model developed to represent the behaviour of parallel multicell converters. The study of this model based on state space equations, and of its eigenvalues. gives a full characterization of the current balancing property. Obtaining an analytical expression between the parameters of the converter and the eigenvalues of the model allows thorough understanding of the balancing property and gives design guidelines. This state space harmonic model also enables straight determination of the steady state operation. Thus the sensitivity of the converter regarding control signal unbalance or shght modification of the component values is finally studied.
\end{abstract}

\section{Notations}

$p \quad$ Nombre de cellules en parallèle

$k \quad$ Indice de numérotation des cellules de commutation

$r \quad$ Nombre d'harmoniques pris en compte dans la modélisation

$z \quad$ Indice de numérotation des harmoniques

$P \quad$ Nombre de valeurs propres complexes

$F_{\text {dec }} \quad$ Fréquence de découpage

$\mathcal{R}_{k} \quad$ Rapport cyclique de la cellule $k$

$\left(^{*}\right)$ Auteur auquel doit être adressée la correspondance (e-mail meynard@leer enseeiht.fr)

$\left({ }^{* *}\right)$ U P R E.S - A. au C N.R.S $\mathrm{N}^{\circ} 5004$

(C) Les Éditions de Physique 1997 
$\mathcal{R} \quad$ Rapport cyclique lorsqu'il est égal dans toutes les cellules

$\Phi_{k} \quad$ Déphasage de la commande de la cellule $k$

$L_{k} \quad$ Inductance de liaison de la cellule $k$

$L \quad$ Inductance de liaison lorsqu'elle est de même valeur dans toutes les cellules

$R_{\text {ch }} \quad$ Résistance de la charge du convertisseur

$L_{\mathrm{ch}} \quad$ Inductance de la charge du convertisseur

$V_{\text {ch }} \quad$ F.e.m. de la charge du convertisseur

$Z_{\mathrm{e}} \quad$ Impédance d'entrée du convertisseur

$Z_{\mathrm{e}}^{n} \quad$ Valeur de l'impédance d'entrée à la fréquence $n F_{\mathrm{dec}}$

$R_{\mathrm{ds}_{\text {on }}} \quad$ Résistance passante d'un interrupteur

$G_{k}^{n} \quad$ Grandeur introduite relative à la cellule $k$ et à l'harmonique de rang $n$

$X \quad$ Matrice d'état du système

$\dot{X} \quad$ Dérivée de la matrice $X$

A Matrice dynamique élémentaire du système

$B \quad$ Matrice de commande élémentaire du système

$A_{\text {ch }} \quad$ Matrice dynamique du système avec prise en compte de la charge

$B_{\text {ch }} \quad$ Matrice de commande du système avec prise en compte de la charge

$\tilde{A}_{\text {ch }} \quad$ Matrice dynamique du système avec prise en compte des $R_{\mathrm{ds}_{\mathrm{on}}}$ et de la charge

$\tilde{B}_{\mathrm{ch}} \quad$ Matrice de commande du système avec prise en compte des $R_{\mathrm{ds}_{\text {on }}}$ et de la charge

$U \quad$ Vecteur d'entrée du système

$M \quad$ Matrice introduite pour la prise en compte de la charge

$I_{p} \quad$ Matrice identité de dimension $p, p$

Fonc $(z)$ Fonction intermédiaire de calcul

$\mathrm{VPR}_{x} \quad x$-ième valeur propre réelle de la matrice dynamique

$\mathrm{VPC}_{x} \quad x$-ième valeur propre complexe de la matrice dynamique

\section{Introduction}

Dans la première partie de l'étude sur les convertisseurs multicellulaires parallèles [1], nous avons décrit le principe de rééquilibrage naturel des courants dans ce type de structure. Il a en particulier été montré que ce phénomène ne peut être expliqué qu'en prenant en compte un ou plusieurs harmoniques. Un tel modèle permettant de caractériser cet équilibrage naturel a été développé et validé.

L'objet de cette deuxième partie concerne donc l'exploitation du modèle obtenu précédemment. Nous essaierons dans un premier temps de caractériser le rééquilibrage naturel en fonction des paramètres du convertisseur (inductances de liaison, filtre d'entrée, ...) et de sa commande (déphasage des signaux de commande entre les cellules, rapport cyclique). Cette modélisation pourra aussi nous permettre de tester la sensibilité du convertisseur vis-à-vis des dissymétries du montage (composants différents selon les cellules) et des erreurs dans la commande (erreur sur les rapports cycliques).

\section{Caractérisation $d u$ rééquilibrage naturel}

2.1. Introduction. - L'analyse du rééquilibrage naturel des courants dans les inductances de liaison et donc dans les cellules de commutation est liée à l'étude des valeurs propres de la matrice dynamique du système d'état représentant le convertisseur. Le calcul numérique de ces valeurs propres permet de caractériser un système donné, mais il se trouve que dans ce 
cas précis, on peut trouver une expression analytique de ces valeurs propres en fonction des paramètres du convertisseur.

2.2. DÉtermination des Valeurs propres par Un Calcul analytique. - Pour déterminer cette relation directe, plusieurs hypothèses simplificatrices ont été nécessaires :

- les inductances de liaison sont égales,

- les rapports cycliques des différentes cellules de commutation sont également identiques,

- le déphasage de la commande de la cellule $k$ est égal à $\Phi_{k}=(k-1) 2 \pi / p$.

À partir des équations d'état du modèle, notre démarche consiste dans un premier temps à trouver une expression analytique permettant de déterminer directement tous les coefficients de la matrice dynamique représentant le convertisseur. Le calcul complet figure en annexe A mais le résultat le plus intéressant est que cette matrice est circulante et à coefficients réels.

La seconde phase du calcul (Annexe B), consiste quant à elle à déterminer les expressions analytiques des valeurs propres en utilisant les propriétés de la matrice dynamique citées précédemment. Le résultat débouche sur trois relations distinctes liées chacunes à des valeurs propres particulières :

- une première relation liée à une valeur propre réelle due aux harmoniques de rang multiple du nombre de cellules en parallèle (Éq. (B.7)),

- une deuxième relation liée à une valeur propre réelle n'existant que si le nombre de cellules est pair (Éq. (B.8)),

- et enfin une troisième relation liée aux valeurs propres complexes de la matrice (E.q. (B.9)).

2.3. Caractérisation de la première relation. - Cette expression est définie par deux termes totalement indépendants :

- Un premier terme dû aux harmoniques de rang multiple du nombre de cellules. Pour un dimensionnement cohérent du convertisseur, ce terme sera très faible car ces harmoniques ne participent pas au rééquilibrage des courants (en effet ces harmoniques existent à l'équilibre et par conséquent, pour limiter les pertes en régıme équilibré, la partie réelle de l'impédance d'entrée sera la plus faible possible à ces fréquences).

- Un autre terme lié aux caractéristiques de la charge. Ce terme correspond exactement à l'inverse de la constante de temps d'un circuit $R, L$ où la résistance vaut $R_{\mathrm{ch}}$ et l'inductance est composée de $L_{\mathrm{ch}}$ en série avec les $p$ inductances de liaison $L$ en parallèle et par conséquent il est l'image de la dynamique de la charge. Ce résultat peut être démontré de manière plus mathématique par le fait que cette valeur propre est associée à un vecteur propre unitaire et donc à la somme des courants dans les inductances de liaison, c'est-à-dire au courant de charge.

Nous pouvons donc affirmer que cette valeur propre caractérise la dynamique de la charge du convertisseur et ne participe pas au rééquilibrage des courants. 

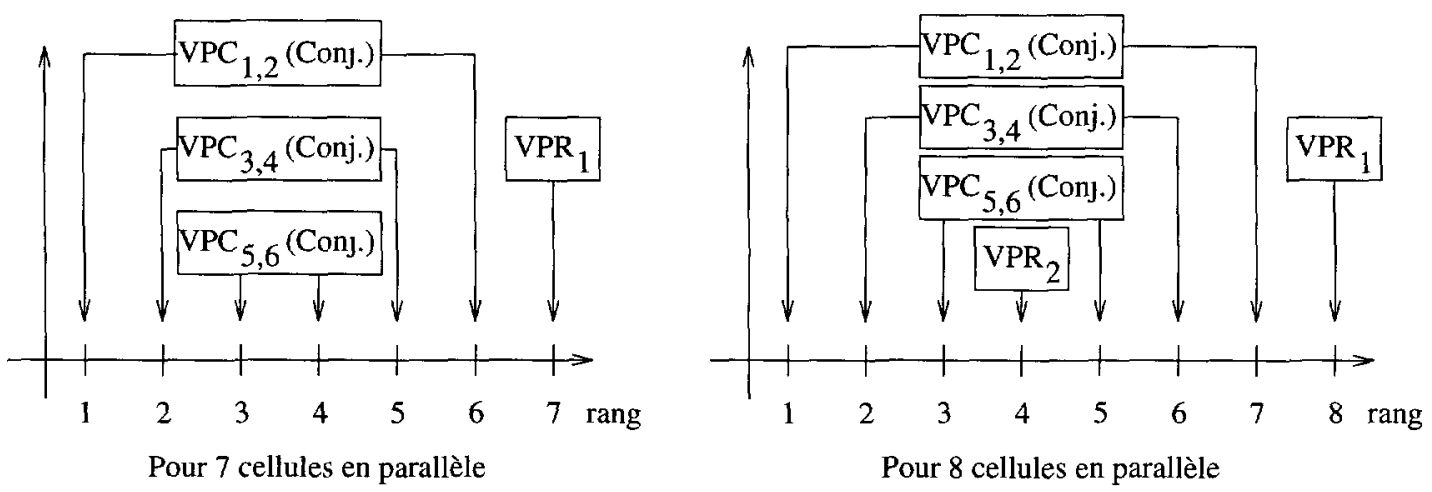

Fig. 1 - Influence des harmoniques sur les valeurs propres.

[Influence of harmonics on eigenvalues.]

2.4. Caractérisation des Deuxième et TRoisième Relations. - Toutes les valeurs propres définies par ces deux relations sont composées de termes dus à tous les harmonıques hormis ceux de rang multiple du nombre de cellules en parallèle En conséquence, elles sont toutes susceptibles d'agir sur le rééquilibrage naturel des courants.

Nous pouvons aussi remarquer que chaque harmonique $z$ influe au maximum sur deux valeurs propres qui sont alors conjuguées Comme, ces deux valeurs propres complexes conjuguées ne définissent qu'une seule dynamique, nous pouvons donc affirmer que toutes les dynamiques de rééquilibrage des courants sont indépendantes entre elles puisqu'elle ne sont pas définies par les mêmes harmoniques.

2.5. Exemples. - Pour illustrer les remarques énoncées dans les deux paragraphes précédents, prenons deux exemples qui permettent de visualiser les différents cas ( $p$ pair ou impair). La figure 1 résume de manière graphique la répartitıon des valeurs propres en fonction du rang des harmoniques compris entre un et le nombre de cellules en parallèle.

Lorsque nous avons sept cellules en parallèle $(p=7)$, les valeurs propres sont de deux types :

- 1 valeur propre réelle ne partıcipant pas au rééquilibrage puisque dépendant de la charge et des harmoniques de rang $z \equiv 0 \bmod [\tau]$,

- 6 valeurs propres complexes conjuguées deux à deux participant au rééquilibrage car dépendant respectivement des harmoniques de rang $z \equiv 1$ et $6 \bmod [7], z \equiv 2$ et $5 \bmod [7]$, $z \equiv 3$ et $4 \bmod [7]$

Lorsque nous avons huit cellules en parallèle $(p=8)$, les valeurs propres sont de trois types :

- I valeur propre réelle ne particıpant pas au rééquilibrage puisque dépendant de la charge et des harmoniques de $\operatorname{rang} z \equiv 0 \bmod [8]$,

- 1 valeur propre réelle participant au rééquilibrage car dépendant des harmoniques de rang $z \equiv 4 \bmod [8]$

- 6 valeurs propres complexes conjuguées deux à deux particıpant au rééquilibrage car dépendant respectivement des harmoniques de rang $z \equiv 1$ et $7 \bmod [8], z \equiv 2$ et $6 \bmod [8]$, $z \equiv 3$ et $5 \bmod [8]$. 
2.6. Conclusion. - Dans ce paragraphe, nous avons déterminé des relations analytıques permettant de calculer directement les valeurs propres à partir des paramètres du convertisseur. Ces expressions analytiques apportent notamment une connaissance profonde des mécanismes de rééquilibrage car elles nous montrent de manière explicite les paramètres du convertisseur qui influent sur ce phénomène. Ainsi le paragraphe suivant s'attachera à étudier l'influence de ces grandeurs afin d'en déduire des critères de dimensionnement.

\section{Influence sur le dimensionnement du convertisseur}

3.1. GÉNÉRALITÉS. - L'objectif de ce paragraphe est de déterminer le rôle des divers paramètres dans le rééquilibrage des courants et d'en quantifier leur influence. Pour cela, nous nous sommes défini les prıncıpes généraux et les hypothèses suivantes :

- la dynamique globale de rééquilibrage des courants dans les inductances de liaison est caractérisée par la dynamique la plus lente du système et par conséquent par la valeur propre la plus faible,

- de plus, pour les valeurs propres complexes, la vitesse de rééquilibrage est caractérisée par la partie réelle de celle-ci, la partie complexe caractérisant quant à elle, les oscillations du rééquilibrage,

- les valeurs propres sont principalement dues aux harmoniques de rang compris entre 1 et $p-1$. En effet, le rôle des rangs supérieurs peut être négligé pour deux raisons :

- dans chaque élément de la somme définissant les valeurs propres, il existe un terme en $1 / z^{2}$ quı rend cet élément négligeable lorsque le rang $z$ augmente,

- de plus, à ces fréquences, l'impédance d'entrée sera très faible (car déjà très faible à $\left.p F_{\mathrm{dec}}\right)$.

Avec cette limitation du nombre d'harmoniques pris en compte, il apparaît que les valeurs propres ne sont plus définies par une somme (Éq. (B.7, B.8, B.9)) mais par un seul terme pour l'éventuelle valeur propre réelle et de deux pour les complexes. Par conséquent. nous adopterons les approximations suivantes pour la suite de l'artıcle

$$
\begin{aligned}
& \forall p \text { entier, } \forall k \in\left\{1, \ldots, \frac{P}{2}\right\} \text {, } \\
& \operatorname{avec}\left\{\begin{array}{l}
P=p-1 \text { si } p \text { impair, } \\
P=p-2 \text { si } p \text { pair, }
\end{array}\right. \\
& \left\{\begin{aligned}
\mathrm{VPR}_{1}= & -\frac{2 p}{L} \Re\left(Z_{\mathrm{e}}^{p}\right) \operatorname{Fonc}(p)-\frac{p R_{\mathrm{ch}}}{L+p L_{\mathrm{ch}}} \\
\mathrm{VPR}_{2}= & -\frac{p}{L} \Re\left(Z_{\mathrm{e}}^{\frac{p}{2}}\right) \operatorname{Fonc}\left(\frac{p}{2}\right) \text { si p pair } \\
\mathrm{VPC}_{2 k-1}= & -\frac{p}{L}\left[\Re\left(Z_{\mathrm{e}}^{k}\right)-\jmath \Im\left(Z_{\mathrm{e}}^{k}\right)\right] \operatorname{Fonc}(k) \\
& -\frac{p}{L}\left[\Re\left(Z_{\mathrm{e}}^{p-k}\right)+\jmath \Im\left(Z_{\mathrm{e}}^{p-k}\right)\right] \operatorname{Fonc}(p-k) \\
\mathrm{VPC}_{2 k}= & \left(\mathrm{VPC}_{2 k-1}\right)^{*}
\end{aligned}\right.
\end{aligned}
$$


3.2. Influence de L'impédance D'entrÉe. - Pour un nombre de cellules $p$ en parallèle, il existe une valeur propre liée à la dynamique de la charge $\mathrm{VPR}_{1}$ et $p-1$ valeurs propres liées au rééquilibrage des courants. Nous avons vu que ces valeurs propres étaient, selon la parité de $p$ soit :

- toutes complexes conjuguées deux à deux si $p$ est impair,

- avec une valeur propre réelle en plus des complexes si $p$ est pair.

Le rééquilibrage des courants n'est assuré que si aucune valeur propre n'est nulle ( $c f .3 .1$ Généralités). Par conséquent, il est souhaitable d'avoir une impédance d'entrée telle que sa partie réelle est :

- nulle pour les harmoniques de rang $p$ de manière à limiter les pertes en régime équilibré,

- non nulle pour les harmoniques de rang $p / 2$ si $p$ est pair pour avoir $\Re\left(\mathrm{VPR}_{2}\right) \neq 0$,

- non nulle pour les harmoniques de rang $k$ ou $p-k$ avec $k \in\left\{1, \ldots, \frac{P}{2}\right\}$, pour avoir $\Re\left(\mathrm{VPC}_{k}\right) \neq 0$ et $\Re\left(\mathrm{VPC}_{p-k}\right) \neq 0$.

Mais, nous pouvons remarquer que le terme $\operatorname{Fonc}(z)$ est en $1 / z^{2}$ et par conséquent il est plus judicieux d'avoir une impédance d'entrée non nulle pour les harmoniques de rang les plus bas de manière à obtenır une valeur propre plus élevée et donc un rééquilibrage plus rapide pour une impédance donnée.

Les remarques évoquées dans ce paragraphe, nous amènent à énoncer un principe indispensable au bon rééquilibrage des courants:

Pour un nombre de cellules en parallèle $p$, il est ındıspensable d'avoır une impédance d'entrée dont la partıe réelle est non nulle pour les $n$ premiers harmonaques avec $n$ égal à partıe entière de $p / 2$. Le rééquilıbrage sera d'autant plus rapıde que la partie réelle de l'ımpédance d'entrée à ces fréquence est forte.

Exemple. - Dans le cas de sept cellules en parallèle et d'une impédance d'entrée de type filtre $R, L, C$ série, pour avoir une dynamique de rééquilıbrage performante il est nécessaire d'avoir trois filtres résonnants à $F_{\mathrm{dec}}, 2 F_{\mathrm{dec}}$ et $3 F_{\mathrm{dec}}$. On aurait pu accorder le deuxième filtre sur $5 F_{\text {dec }}$ au lieu de $2 F_{\text {dec }}$ mais pour une même valeur de résistance, la valeur propre liée à ce filtre aurait été $25 / 4 \simeq 6$ fois plus faible.

3.3. INFLUENCE DE LA VALEUR DU RAPPORT CYCLIQUE - Dans la définition des valeurs propres (Éq. (1)), le rapport cyclique n'intervient que dans le terme Fonc( $z$ ). Ce terme en sinus est donc susceptible d'être égal à zéro pour certaines valeurs de rapport cyclique entraînant une possible annulation de la valeur propre. Donc, l'influence du rapport cyclique est primordiale dans le rééquilibrage des courants et fera l'objet d'une étude approfondie.

Dans le cas d'un nombre pair de cellule, la valeur propre réelle $\mathrm{VPR}_{2}$ n'est due qu'à l'harmonique de rang $p / 2$ et par conséquent lorsque $\operatorname{Fonc}(p / 2)$ est nul, le rééquilibrage n'est plus assuré et cela quel que soit l'impédance d'entrée. Par exemple, pour 6 cellules en parallèle, nous avons Fonc $(3)=0$ pour $\mathcal{R}=0 ; 0,33 ; 0,66$ et 1 .

Les valeurs propres complexes $\mathrm{VPC}_{2 k}$ et $\mathrm{VPC}_{2 k}$ sont quant à elles définies par deux termes liés aux harmoniques $k$ et $p-k$. Ainsi, s'il existe une valeur de rapport cyclique telle que Fonc $(k)=$ Fonc $(p-k)=0$, alors il n'y a pas de rééquilıbrage possible. Il est intéressant de noter que cette double égalité n'est pas possible si $p$ est un nombre premier ( $c f$. Annexe $C$ ). Cette condition n'est pas suffisante si l'on se place dans le cas limite défini dans le paragraphe 

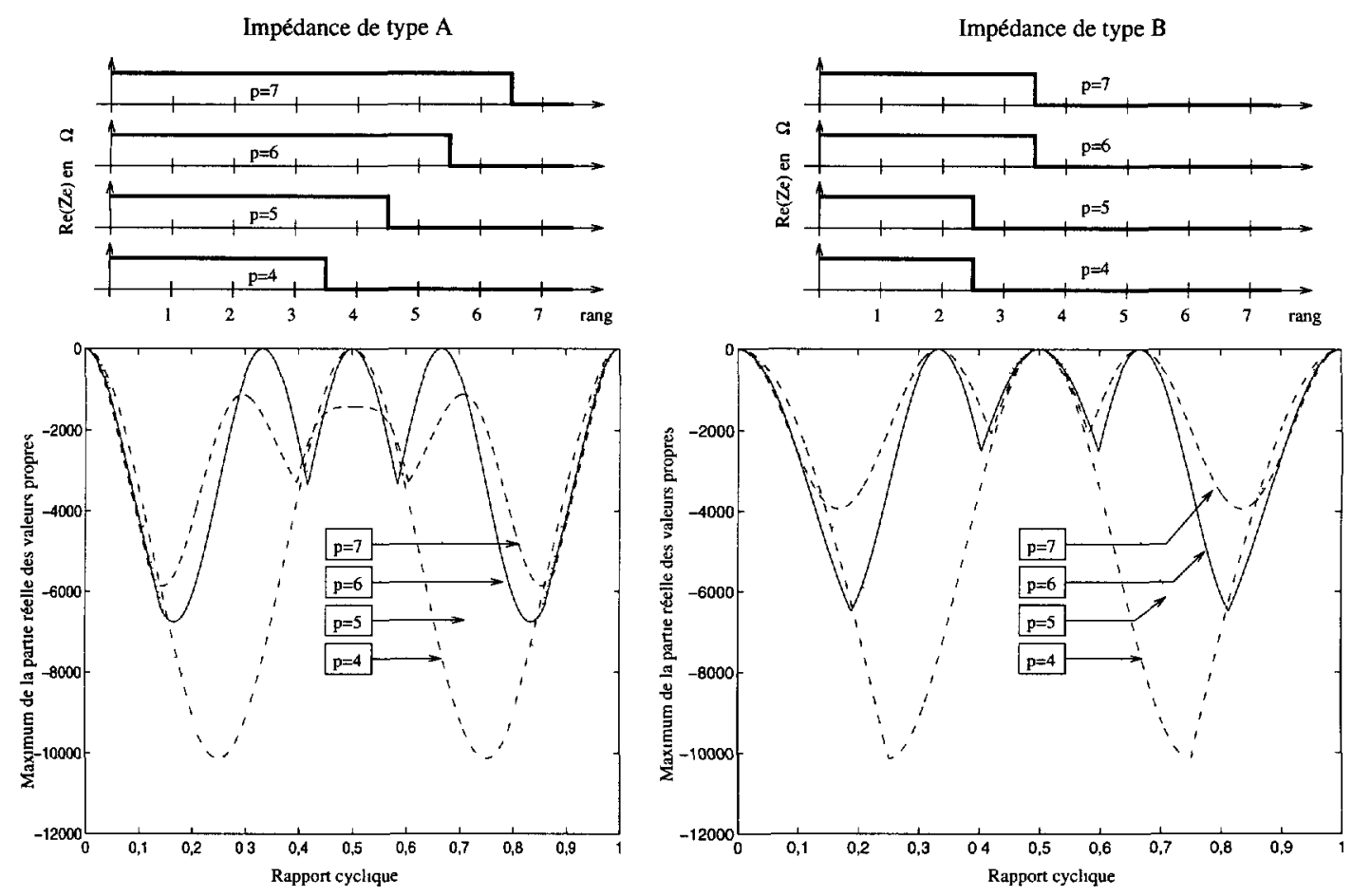

Fig. 2. - Maximum des valeurs propres en fonction du rapport cyclique [Highest value of eigenvalues versus duty cycle.]

précédent $\left(\Re\left(Z_{\mathrm{e}}^{z}\right)=0\right.$ avec $z \in\{n+1, \ldots, p-1\}$ ), les valeurs propres complexes ne sont définies que par un seul terme et l'on se retrouve dans le même cas que pour la valeur propre réelle.

Les courbes obtenues par la méthode directe de calcul des valeurs propres, présentées figure 2 , montrent la variation du maximum des valeurs propres du système pour différentes valeurs du nombre de cellules (avec des inductances de liaison de $500 \mu \mathrm{H}$ ) et pour deux types d'impédance d'entrée (type A ou B).

Ces deux extrêmes impossibles à réaliser dans la pratique montrent toutefois vers quel type d'impédance d'entrée il faudra tendre afin d'obtenir les caractéristiques désirées. Dans les quatre exemples présentés, nous pouvons illustrer tous les cas envisagés dans notre étude .

- quel que soit le nombre de cellules en parallèle et pour les deux types d'impédance, la valeur propre maximale est nulle pour $\mathcal{R}=0$ et $\mathcal{R}=1$, phénomène tout à fait normal puisque pour ces valeurs de rapport cyclique $\operatorname{Fonc}(z)=0$ quel que soit $z$,

- pour $p=4$, quel que soit l'impédance du filtre, la valeur propre réelle liée à l'harmonique 2 s'annule pour $\mathcal{R}=0,5$,

- pour $p=5$, il n'y a pas de problème pour l'impédance de type $A$, mais par contre, pour le type $B$, la valeur propre liée aux harmoniques 2 et 3 est nulle pour $\mathcal{R}=0,5$ car Fonc $(2)=0$ et $Z_{\mathrm{e}}^{3}=0$,

- pour $p=6$, nous avons pour les deux sortes d'impédance, la même allure de courbe car les passages par zéro sont uniquement dus à $\operatorname{Fonc}(z)$. En effet, la valeur propre réelle 
liée à $z=3$ est nulle pour $\mathcal{R}=0,33$ et $\mathcal{R}=0,66$ car Fonc $(3)=0$ et la valeur propre complexe liée à $z=2$ et $z=4$ est nulle pour $\mathcal{R}=0,5 \operatorname{car} \operatorname{Fonc}(2)=\operatorname{Fonc}(4)=0$,

- pour $p=7$. nous pouvons faire les mêmes remarques que pour $p=5$, sauf que dans le type $B$, la valeur propre maximale est nulle pour $\mathcal{R}=0,5$ car Fonc $(2)=0$ et $Z_{\mathrm{e}}^{5}=0$ et pour $\mathcal{R}=0,33$ et $\mathcal{R}=0,66 \operatorname{car} \operatorname{Fonc}(3)=0$ et $Z_{\mathrm{e}}^{4}=0$.

Ces derniers résultats nous montrent la difficulté d'obtenir un bon rééquilibrage quel que soit le rapport cyclique. Dans un fonctionnement de type onduleur où le rapport cyclique est continuellement variable, la consıdération de ces valeurs critiques de rapport cyclıque où le rééquilibrage est inexistant n'est pas indispensable si l'on suppose que le convertisseur ne peut pas subir de brusques déséquilibres. Par contre, dans le cas d'un fonctionnement de type hacheur, ces zones critiques sont inacceptables et par conséquent nous pouvons énoncer le principe suivant :

Dans un fonctionnement du convertisseur multicellulaıre parallèle en mode hacheur, le nombre de cellules de commutation en parallèle devra être un nombre premier. De plus, la partıe réelle de l'impédance d'entrée devra être non nulle sur les $p-1$ premiers harmonıques.

3 4. INFLUENCE DES AUTRES GRANDEURS - Les autres paramètres inclus dans les calcul des valeurs propres sont les inductances de liaison et la partie imaginaire des valeurs propres complexes.

Pour le premier terme, d'un point de vue rééquilibrage nous voyons que nous avons intérêt à avoir des inductances les plus faibles possibles de manière à obtenir une dynamique importante. Cependant, le rôle de ces inductances est de limiter les ondulations de courants dans les cellules de commutation et par conséquent c'est ce critère que nous prendrons en considération pour le choix des valeurs d'inductances en tenant compte du fait qu'elles ne doivent pas avoir de valeurs démesurées

En ce qui concerne la partie imaginaire des valeurs propres, on se rend compte qu'elle provoque des oscillations dans la dynamique de rééquilibrage et l'intérêt est donc de les limiter au maximum. Cependant, elle est générée par la partie imaginaire de l'impédance d'entrée aux multiples de la fréquence de découpage et donc très difficile à limiter puisque dans le cas de filtres $R, L, C$, hors de la résonance, cette impédance est fortement inductive ou capacitive.

3.5 Conclusion, - L'étude du rééquilibrage naturel des courants des inductances de liaison d'après la modélisation effectuée dans la première partie de l'article nous a conduit à étudier la variation des valeurs propres en fonction des paramètres du convertisseur et de sa commande. Pour avoir un rééquilibrage assuré. 11 est indispensable d'avoir toutes les valeurs propres non nulles car la constante de temps du rééquilibrage global de tous les courants est définie à partir de la valeur propre la plus faible. Par conséquent, ll est par exemple nécessaire d'avoir la partie réelle de l'impédance d'entrée non nulle jusqu'à au moins l'harmonique de rang égal à la partie entière de $p / 2$. De plus, les valeurs propres ont toutes un terme en $\sin (z \pi \mathcal{R})$ et par conséquent, pour certaines valeurs de rapport cyclique, le rééquilibrage sera très lent voire inexistant.

\section{Sensibilité du convertisseur aux perturbations}

4.1. IntRoduction. - Le chapitre précédent nous a permis de quantifier les dynamiques de rééquilibrage en fonctıon des divers paramètres du convertisseur. Cependant, les simulations ayant servi à valider le modèle [1] montrent qu'en cas d'erreur sur la commande (rapport cyclique différent selon les cellules de commutation) ou bien en cas de différences entre les 
$R_{\mathrm{ds}_{\mathrm{on}}}$ des composants des diverses cellules, il existe un déséquilibre des courants en régime permanent que nous nous proposons de caractériser.

Pour cela, nous nous placerons dans le cas d'un convertisseur à quatre cellules de commutation en parallèle et possédant une impédance d'entrée de la forme :

- $\Re\left(Z_{\mathrm{e}}\right)=$ cste et $\Im\left(Z_{\mathrm{e}}\right)=0$ pour les harmoniques de rang 1 et 2 ,

- $\Re\left(Z_{\mathrm{e}}\right)=0$ et $\Im\left(Z_{\mathrm{e}}\right)=0$ pour tous les autres harmoniques.

Nous avons choisi ce type d'impédance d'entrée car c'est la caractéristique minımale qu'elle doit avoir pour assurer le rééquilibrage naturel des courants ( $c f .3 .2$. Influence de l'impédance d'entrée).

4.2. Sensibilité auX erreurs de Rapport CyClique. - Nous avons vu précédemment qu'une condition importante pour la commande des convertisseurs multicellulaires est d'avoir un rapport cyclique égal pour toutes les cellules de commutation. En pratique, cette égalité parfarte étant irréalisable, nous allons étudier l'influence d'une erreur sur le régime permanent des courants dans les inductances de liaison en fonction de la valeur de la partie réelle de l'impédance d'entrée.

Les résultats présentés sur la figure 3 ont été déterminés pour un rapport cyclique de 0,8 avec une erreur .

- de $-1 \%$ pour la cellule 1 ,

- de $-2 \%$ pour la cellule 2 ,

- de $0,5 \%$ pour la cellule 3 ,

- de $1 \%$ pour la cellule 4 .

Ces résultats montrent la difficulté de mettre en parallèle des cellules de commutation sans impédance d'entrée $\left(Z_{\mathrm{e}}=0\right)$. En effet, pour une erreur de rapport cyclique de quelques pourcent, le déséquilibre en régime permanent est très important. Heureusement, la présence de cette impédance permet de réduire nettement le déséquilibre. Sur notre exemple, il passe de $120 \mathrm{~A}$ sans impédance à $20 \mathrm{~A}$ pour $4 \Omega$.

4.3. Sensibilité AuX DiffÉRences ENTRE LES $R_{\mathrm{ds}_{o_{n}}}$. - Les techniques de fabrication actuelles des composants de puissance ne permettent pas de garantir une parfaite homogénéité des caractéristiques. Dans le cas de Mosfet, leurs $R_{\text {ds }_{\text {on }}}$ peuvent différer de plus de $30 \%$.

Pour illustrer l'influence de ces imperfections, nous avons calculé le régime permanent des courants dans les inductances de liaison en fonction des valeurs de la partie réelle de l'impédance d'entrée pour les valeurs de $R_{\mathrm{ds}_{\mathrm{on}}}$ suivantes

- cellule $1: R_{\mathrm{ds}_{\mathrm{on}}}=80 \mathrm{~m} \Omega$,

- cellule $2: R_{\mathrm{ds}_{\mathrm{on}}}=95 \mathrm{~m} \Omega$,

- cellule $3: R_{\mathrm{ds}_{\mathrm{on}}}=110 \mathrm{~m} \Omega$,

- cellule $4: R_{\mathrm{ds}_{\mathrm{on}}}=100 \mathrm{~m} \Omega$.

Les résultats obtenus figure 4 montrent l'intérêt de l'impédance d'entrée pour diminuer le déséquilibre des courants en régime permanent. En effet, sans cette impédance $\left(Z_{\mathrm{e}}=0\right)$, le déséquilibre est directement proportionnel aux différences entre les $R_{\mathrm{ds}_{\mathrm{on}}}$. Par contre, en présence de celle-ci. les résultats sont tout à fait satisfaisant puisque le déséquilibre est encore fortement réduit. 


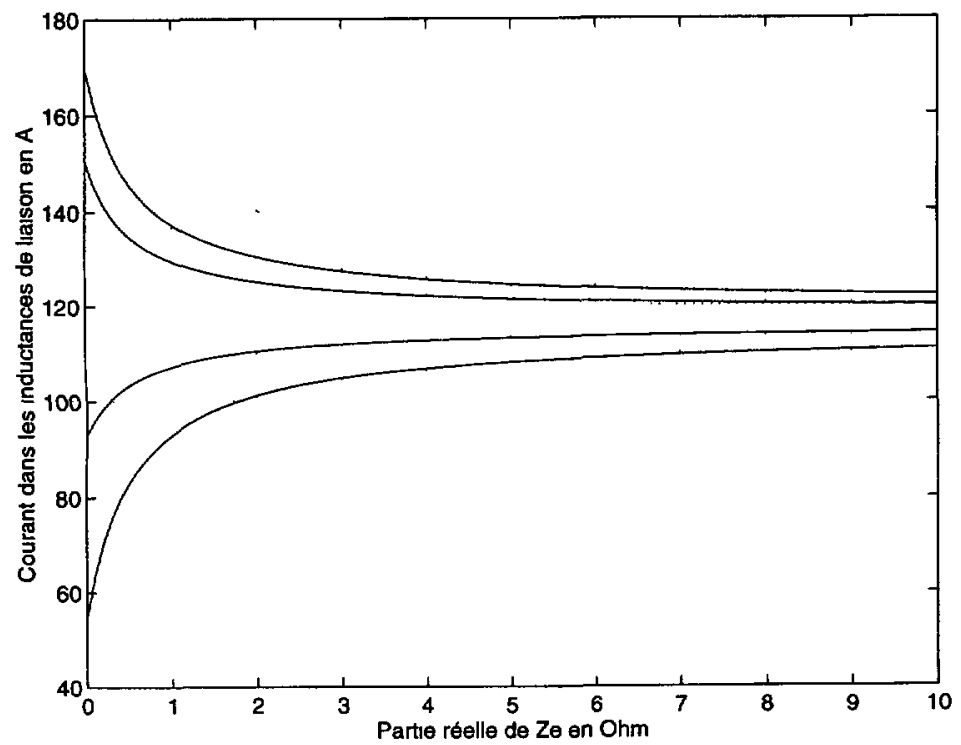

Fig. 3. - Courants en régime permanent pour des déséquilibres de rapport cyclique. [Steady state current for unbalanced duty cycle]

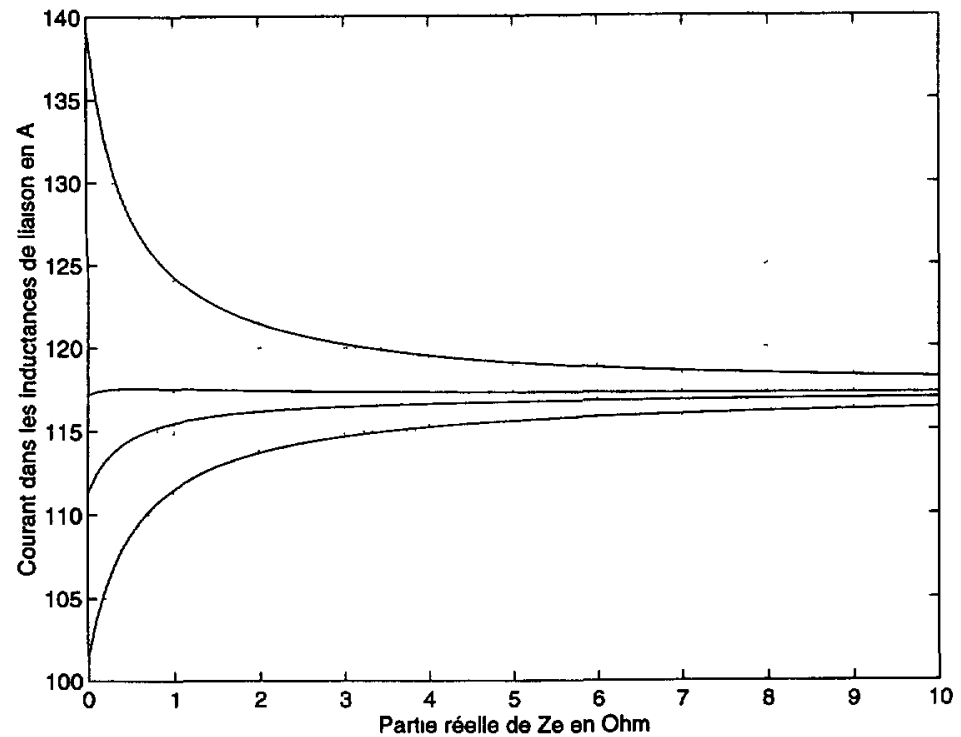

Fig. 4. - Courants en régime permanent pour des $R_{\mathrm{ds}_{\mathrm{on}}}$ différentes.

[Steady state current for different $R_{\mathrm{ds}_{\mathrm{on}}}$.]

4.4. Conclusion. - L'intérêt de ce dernier paragraphe était de montrer l'influence de l'impédance d'entrée sur le régime permanent d'un convertisseur multicellulaire parallèle.

Ainsi nous avons pu vérifier, que cette impédance d'entrée jouait un rôle prépondérant dans la 

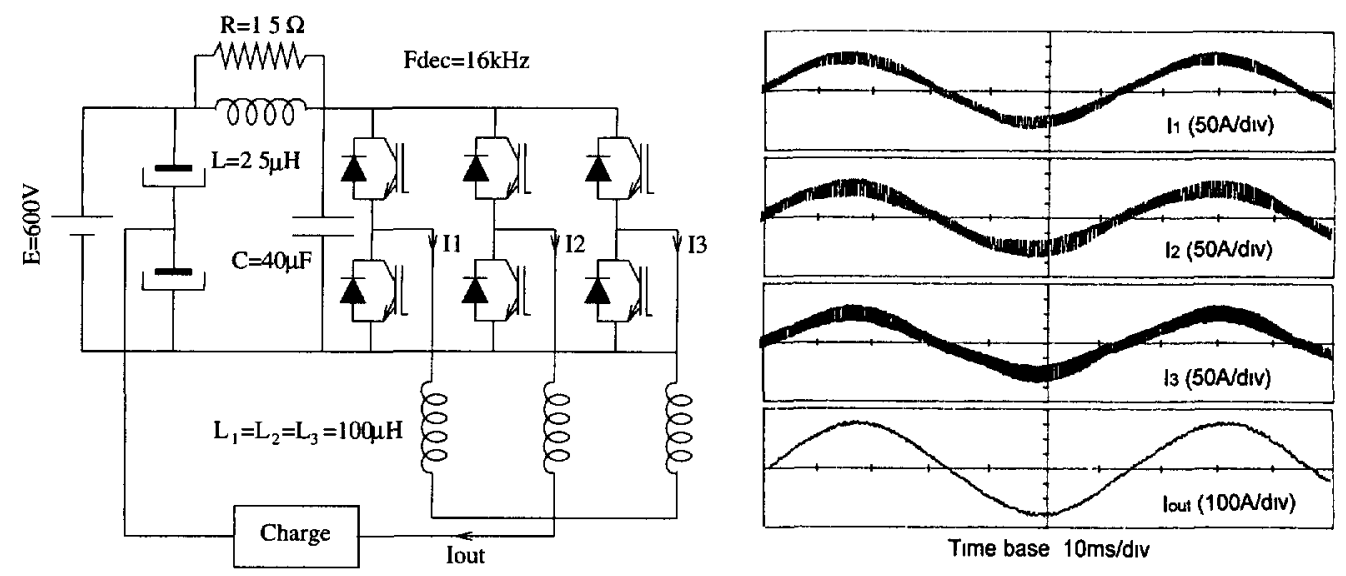

Fig. 5. - Schéma et résultats du montage expérımental.

[Scheme and results of experimental converter.]

valeur finale du courant dans les inductances de liaison et permettait de dimmnuer très fortement les déséquilibres dus à des erreurs de commande ou à des dissymétries de la structure.

\section{Exemple de réalisation}

Afin de mieux appréhender la définition de l'impédance d'entrée, nous proposons de montrer les résultats expérimentaux obtenus avec un onduleur à point milieu capacitif réalisé avec des IGBTs de calibre $1200 \mathrm{~V}-200 \mathrm{~A}$.

Nous pouvons remarquer que l'impédance d'entrée $Z_{\mathrm{e}}$ définie dans les paragraphes précédents n'est en fait que le filtre d'entrée du convertisseur que nous avons dimensionné de telle sorte que sa fréquence de résonance soit à la fréquence de découpage de l'onduleur. Ainsi, nous avons bien une impédance d'entrée conforme à nos besoins (forte valeur à la fréquence de découpage et valeur négligeable à trois fois celle-ci comme le montre la figure 6).

Avec cette caractéristique d'impédance, il est intéressant de noter que la puissance dissipée dans la résistance du filtre n'est pas préjudiciable pour le rendement du convertisseur et pas pénalisable pour la réalisation de la résistance. En effet, au cours des essais réalisés sous une puissance de $45 \mathrm{kVA}$, la résistance dimensionnée pour $100 \mathrm{~W}$ n'a pas chauffé de manière significative.

\section{Conclusion}

La mise en œuvre d'un modèle mathématique représentant de manière fidèle le comportement d'un convertisseur de type multicellulaire parallèle était destinée à mieux appréhender son fonctionnement et plus particulièrement le phénomène de rééquilibrage naturel des courants dans les inductances de liaison, qui jusque là, n'était pas bien maîtrisé.

Les résultats obtenus sont tout à fait intéressant, puisque d'un point de vue dynamique nous avons réussi à quantifier les constantes de temps de rééquilibrage en fonction des paramètres de la structure et notamment de l'impédance d'entrée. À partir de ces équations, nous avons pu expliquer le rééquilibrage et ainsi déduire des critères de dimensionnement. Ce modèle nous a aussi permis d'étudier le fonctionnement du convertisseur en régime statique. Nous avons 


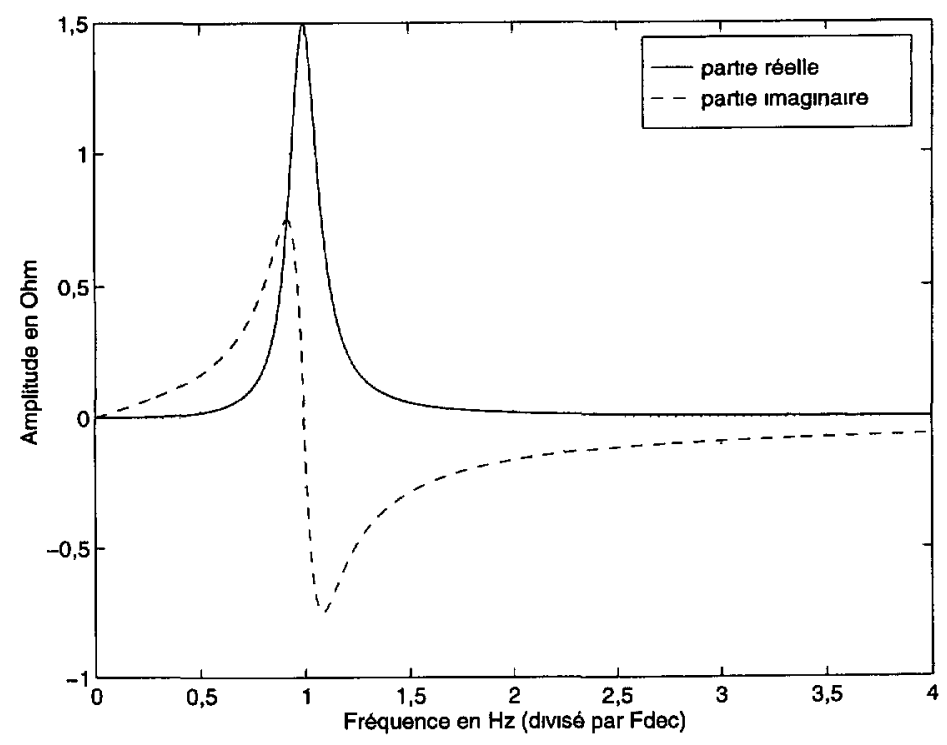

Fig 6. - Impédance du filtre d'entrée en fonction de la fréquence

[Input filter impedance versus frequency]

pu vérifier l'ımportance de l'impédance d'entrée puisqu'elle influe directement sur la valeur du régime permanent et permet de réduire fortement les déséquilibres.

\section{Annexe A}

\section{Calcul des éléments de la matrice dynamique}

A.1. Matrice dynamique sans prise en compte de la charge (Matrice $A$ ). - Dans ce paragraphe nous allons calculer les coefficients de la matrice dynamique $A$ issue de l'étude réalisée dans la première partie de l'article [1] dont nous rappelons ci-dessous la forme :

$$
A=-2 \Re\left(\left[\begin{array}{lll}
\frac{1}{L_{1}}\left(G_{1}^{1}\right)^{*} & \cdots & \frac{1}{L_{1}}\left(G_{1}^{r}\right)^{*} \\
\vdots & \ddots & \vdots \\
\frac{1}{L_{p}}\left(G_{p}^{1}\right)^{*} & \cdots & \frac{1}{L_{p}}\left(G_{p}^{r}\right)^{*}
\end{array}\right]\left[\begin{array}{lll}
Z_{\mathrm{e}}^{1} G_{1}^{1} & \cdots & Z_{\mathrm{e}}^{1} G_{p}^{1} \\
\vdots & \ddots & \\
Z_{\mathrm{e}}^{r} G_{1}^{r} & \cdots & Z_{\mathrm{e}}^{r} G_{p}^{r}
\end{array}\right]\right)
$$

Dans notre cas, toutes les cellules de commutation ont la même influence sur la charge et par conséquent nous prendrons donc $\forall k \in\{1, \ldots, p\}, L_{k}=L$. Ainsi nous pouvons écrire .

$$
A=-\frac{2}{L} \Re\left[\begin{array}{lll}
\sum_{z=1}^{r} Z_{\mathrm{e}}^{z} G_{1}^{z}\left(G_{1}^{z}\right)^{*} & \cdots & \sum_{z=1}^{r} Z_{\mathrm{e}}^{z} G_{p}^{z}\left(G_{1}^{z}\right)^{*} \\
\vdots & \ddots & \vdots \\
\sum_{z=1}^{r} Z_{\mathrm{e}}^{z} G_{1}^{z^{-1}}\left(G_{p}^{z}\right)^{*} & \ldots & \sum_{z=1}^{r} Z_{\mathrm{e}}^{z} G_{p}^{z}\left(G_{p}^{z}\right)^{*}
\end{array}\right] .
$$


Il apparaît dans l'équation (A.2) que chaque élément de la matrice $A$ de la ligne $\imath$ et de la colonne $l \jmath$ peut s'écrire sous la forme:

$$
A(\imath 2, \jmath \jmath)=-\frac{2}{L} \Re\left(\sum_{z=1}^{r} Z_{\mathrm{e}}^{z} G_{\jmath \jmath}^{z}\left(G_{\imath \imath}^{z}\right)^{*}\right) .
$$

Pour les mêmes raisons que pour les inductances de liaison, les rapports cycliques $\mathcal{R}_{k}$ sont tous égaux et valent $\mathcal{R}$. De plus, l'angle de déphasage des signaux de commande de la cellule $k$ vaut $\Phi_{k}=(k-1) 2 \pi / p$. Ainsi d'après les équations décrites dans [1] nous pouvons écrire :

$$
G_{k}^{z}=\frac{1}{z \pi} \sin (z \pi \mathcal{R}) \exp \left(j \frac{2 \pi}{p} z(k-1)\right) .
$$

En remplaçant dans l'équation (A.3), $G_{k}^{z}$ par sa valeur, nous obtenons directement les éléments de la matrice $A$ en fonction des paramètres du convertisseur :

$$
A(\imath, j \jmath)=-\frac{\dot{2}}{L} \Re\left(\sum_{z=1}^{r} Z_{\mathrm{e}}^{z}\left(\frac{\sin (z \pi \mathcal{R})}{z \pi}\right)^{2} \exp \left(\jmath \frac{2 \pi z}{p}(\jmath \jmath-\imath \imath)\right)\right) .
$$

Nous pouvons remarquer d'après l'expression des éléments de la matrice $A$ (Éq. (A.5)) qu'elle est circulante à coefficients réels.

A.2 Matrice dynamique avec prise en compte de la charge (Matrice $A_{\mathrm{ch}}$ ). La matrice $A_{\mathrm{ch}}$ calculé dans [1], en utilisant la simplification liée à l'égalité des inductances de liaison, s'écrire sous la forme :

$$
A_{\mathrm{ch}}=M^{-1}\left(A-\frac{R_{\mathrm{ch}}}{L} J_{p}\right)
$$

avec :

$$
M=I_{p}+\frac{R_{\mathrm{ch}}}{L} J_{p} \text { ou } I_{p}=\left[\begin{array}{ccc}
1 & 0 & 0 \\
0 & \ddots & 0 \\
0 & 0 & 1
\end{array}\right] \text { et } J_{p}=\left[\begin{array}{ccc}
1 & \cdots & 1 \\
\vdots & \ddots & \vdots \\
1 & \cdots & 1
\end{array}\right]
$$

Avec cette définition de $M$, la matrice inverse $M^{-1}$ vaut :

$$
M^{-1}=I_{p}-\frac{L_{c h}}{L+p L_{\mathrm{ch}}} J_{p}
$$

On peut ainsı calculer les éléments de $A_{\text {ch }}$ à partir de ceux de $A$ :

$$
A_{\mathrm{ch}}(\imath \imath, \jmath \jmath)=A(\imath, \jmath \jmath)-\frac{R_{\mathrm{ch}}}{L}+\frac{L_{\mathrm{ch}}}{L+p L_{\mathrm{ch}}}\left(\frac{p R_{\mathrm{ch}}}{L}-\sum_{x=1}^{p} A(x, \jmath \jmath)\right) \text {. }
$$

Comme la matrice $A$ est circulante à coefficients réels, il en est de même pour $A_{\text {ch }}$ puisque l'on rajoute uniquement une même constante réelle à tous les éléments de $A(\imath \imath, \jmath \jmath)$ pour obtenir $A_{\mathrm{ch}}(\imath \imath . \jmath \jmath)$.

\section{Annexe B}

\section{Calcul des valeurs propres}

Le calcul des valeurs propres est réalisé à partir des équatıons fournies par le logiciel Mathematıca dans le cas de matrice circulante à coefficients réels de dimension $p$. Les valeurs propres réelles seront notées $\mathrm{VPR}_{x}$ et les valeurs propres complexes $\mathrm{VPC}_{x}$. 
CAS DE $P=3$. - Nous avons dans ce cas une valeur propre réelle $\mathrm{VPR}_{1}$ et deux complexes $\mathrm{VPC}_{1}$ et $\mathrm{VPC}_{2}$ :

$$
\left\{\begin{aligned}
\mathrm{VPR}_{1} & =A_{\mathrm{ch}}(1,1)+A_{\mathrm{ch}}(1,2)+A_{\mathrm{ch}}(1,3) \\
\mathrm{VPC}_{1} & =A_{\mathrm{ch}}(1,1)-\frac{A_{\mathrm{ch}}(1,2)+A_{\mathrm{ch}}(1,3)}{2}+\jmath \frac{\sqrt{3}}{2}\left(A_{\mathrm{ch}}(1,2)-A_{\mathrm{ch}}(1,3)\right) \\
\mathrm{VPC}_{2} & =\left(\mathrm{VPR}_{1}\right)^{*}
\end{aligned}\right.
$$

Avec la définition de $A_{\mathrm{ch}}(\imath \imath, \jmath \jmath)$ (Éq. (A.9)), nous pouvons calculer les valeurs propres de la matrice dynamique :

$$
\left\{\begin{aligned}
\mathrm{VPR}_{1}= & -\frac{2}{L} \Re\left(\sum_{z=1}^{r}\left\{Z_{\mathrm{e}}^{z}\left(\frac{\sin (z \pi \mathcal{R})}{z \pi}\right)^{2}\left(1+\exp \left(\jmath \frac{2 \pi z}{3}\right)+\exp \left(\jmath \frac{4 \pi z}{3}\right)\right)\right\}\right) \\
& \left.\left.-\frac{3 R_{\mathrm{ch}}}{L+3 L_{\mathrm{ch}}}\right)\right\} \\
\mathrm{VPC}_{1}= & -\frac{2}{L} \Re\left(\sum_{z=1}^{r}\left\{Z_{\mathrm{e}}^{z}\left(\frac{\sin (z \pi \mathcal{R})}{z \pi}\right)^{2}\left(1-\frac{\exp \left(\jmath \frac{2 \pi z}{3}\right)+\exp \left(\jmath \frac{4 \pi z}{3}\right)}{2}\right)\right\}\right) \\
& +\jmath \frac{\sqrt{3}}{L} \Re\left(\sum_{z=1}^{r}\left\{Z_{\mathrm{e}}^{z}\left(\frac{\sin (z \pi \mathcal{R})}{z \pi}\right)^{2}\left(\exp \left(\jmath \frac{2 \pi z}{3}\right)-\exp \left(\jmath \frac{4 \pi z}{3}\right)\right)\right\}\right) \\
\mathrm{VPC}_{2}= & \left(\mathrm{VPC}_{1}\right)^{*}
\end{aligned}\right.
$$

Nous pouvons remarquer que chaque valeur propre est une somme de termes liés à un harmonique $z$. En étudiant ces termes un par un, des simplifications importantes apparaîssent puisque :

\begin{tabular}{|c|c|c|c|}
\hline & $z \equiv 0 \bmod [3]$ & $z \equiv 1 \bmod [3]$ & $z \equiv 2 \bmod [3]$ \\
\hline $1+\mathrm{e}^{j \frac{2 \pi z}{3}}+\mathrm{e}^{j \frac{4 \pi z}{3}}$ & 3 & 0 & 0 \\
\hline $1-\frac{\mathrm{e}^{\frac{2 \pi z}{3}}+\mathrm{e}^{j \frac{4 \pi z}{3}}}{2}$ & 0 & $3 / 2$ & $3 / 2$ \\
\hline $\mathrm{e}^{\jmath \frac{2 \pi z}{3}}-\mathrm{e}^{\jmath \frac{4 \pi z}{3}}$ & 0 & $3 \sqrt{3}$ & $-3 \sqrt{3}$ \\
\hline
\end{tabular}

Ainsi les valeurs propres du système pour $p=3$ sont de la forme :

$$
\left\{\begin{aligned}
\mathrm{VPR}_{1}= & -\frac{6}{L} \sum_{z \equiv 0 \bmod [3]}^{r}\left\{\Re\left(Z_{\mathrm{e}}^{z}\right)\left(\frac{\sin (z \pi \mathcal{R})}{z \pi}\right)^{2}\right\}-\frac{3 R_{\mathrm{ch}}}{L+3 L_{\mathrm{ch}}} \\
\mathrm{VPC}_{1}= & -\frac{3}{L} \sum_{z \equiv 1 \bmod [3]}^{r}\left\{\left[\Re\left(Z_{\mathrm{e}}^{z}\right)-\jmath \Im\left(Z_{\mathrm{e}}^{z}\right)\right]\left(\frac{\sin (z \pi \mathcal{R})}{z \pi}\right)^{2}\right\} \\
& -\frac{3}{L} \sum_{z \equiv 2 \bmod [3]}^{r}\left\{\left[\Re\left(Z_{\mathrm{e}}^{z}\right)+\jmath \Im\left(Z_{\mathrm{e}}^{z}\right)\right]\left(\frac{\sin (z \pi \mathcal{R})}{z \pi}\right)^{2}\right\} \\
\mathrm{VPC}_{2}= & \left(\mathrm{VPC}_{1}\right)^{*}
\end{aligned}\right.
$$


CAS DE $P=4$. - Dans ce cas, il apparaît quatre valeurs propres, deux réelles $V P R_{1}$ et $V_{P R}$ et deux complexes $\mathrm{VPC}_{1}$ et $\mathrm{VPC}_{2}$ telles que :

$$
\left\{\begin{aligned}
\mathrm{VPR}_{1} & =A_{\mathrm{ch}}(1,1)+A_{\mathrm{ch}}(1,2)+A_{\mathrm{ch}}(1,3)+A_{\mathrm{ch}}(1,4) \\
\mathrm{VPR}_{2} & =A_{\mathrm{ch}}(1,1)-A_{\mathrm{ch}}(1,2)+A_{\mathrm{ch}}(1,3)-A_{\mathrm{ch}}(1,4) \\
\mathrm{VPC}_{1} & =\left(A_{\mathrm{ch}}(1,1)-A_{\mathrm{ch}}(1,3)\right)+j\left(A_{\mathrm{ch}}(1,2)-A_{\mathrm{ch}}(1,4)\right) \\
\mathrm{VPC}_{2} & =\left(\mathrm{VPC}_{1}\right)^{*}
\end{aligned}\right.
$$

Comme précédemment, ces valeurs propres peuvent être calculées en fonction des caractéristiques du convertisseur :

$$
\left\{\begin{aligned}
\mathrm{VPR}_{1}= & -\frac{2}{L} \Re\left(\sum _ { z = 1 } ^ { r } \left\{Z _ { \mathrm { e } } ^ { z } ( \frac { \operatorname { s i n } ( z \pi \mathcal { R } ) } { z \pi } ) ^ { 2 } \left(1+\exp \left(\jmath \frac{\pi z}{2}\right)+\exp (\jmath \pi z)\right.\right.\right. \\
& \left.\left.\left.+\exp \left(j \frac{3 \pi z}{2}\right)\right)\right\}\right)-\frac{4 R_{\mathrm{ch}}}{L+4 L_{\mathrm{ch}}} \\
\mathrm{VPR}_{2}= & -\frac{2}{L} \Re\left(\sum _ { z = 1 } ^ { r } \left\{Z _ { \mathrm { e } } ^ { z } ( \frac { \operatorname { s i n } ( z \pi \mathcal { R } ) } { z \pi } ) ^ { 2 } \left(1-\exp \left(j \frac{\pi z}{2}\right)+\exp (j \pi z)\right.\right.\right. \\
& \left.\left.\left.-\exp \left(j \frac{3 \pi z}{2}\right)\right)\right\}\right) \\
\mathrm{VPC}_{1}= & -\frac{2}{L} \Re\left(\sum_{z=1}^{r}\left\{Z_{\mathrm{e}}^{z}\left(\frac{\sin (z \pi \mathcal{R})}{z \pi}\right)^{2}(1-\exp (j \pi z))\right\}\right) \\
& -j \frac{2}{L} \Re\left(\sum_{z=1}^{r}\left\{Z_{\mathrm{e}}^{z}\left(\frac{\sin (z \pi \mathcal{R})}{z \pi}\right)^{2}\left(\exp \left(j \frac{\pi z}{2}\right)-\exp \left(j \frac{3 \pi z}{2}\right)\right)\right\}\right) \\
\mathrm{VPC}_{2}= & \left(\mathrm{VPC}_{1}\right)^{*}
\end{aligned}\right.
$$

Comme dans le cas $p=3$, les simplifications suivantes apparaîssent dans les équation (B.5) :

\begin{tabular}{|c|c|c|c|c|}
\hline & $z \equiv 0 \bmod [4]$ & $z \equiv 1 \bmod [4]$ & $z \equiv 2 \bmod [4]$ & $z \equiv 3 \bmod [4]$ \\
\hline $1+\mathrm{e}^{3 \frac{\pi z}{2}}+\mathrm{e}^{\jmath \pi z}+\mathrm{e}^{\jmath \frac{3 \pi z}{2}}$ & 4 & 0 & 0 & 0 \\
\hline $1-\mathrm{e}^{j \frac{\pi z}{2}}+\mathrm{e}^{j \pi z}-\mathrm{e}^{3 \frac{3 \pi z}{2}}$ & 0 & 0 & 4 & 0 \\
\hline $1-\mathrm{e}^{\jmath \pi z}$ & 0 & 2 & 0 & 2 \\
\hline $\mathrm{e}^{\jmath \frac{\pi z}{2}}-\mathrm{e}^{3 \frac{3 \pi z}{2}}$ & 0 & $2 j$ & 0 & $-2 j$ \\
\hline
\end{tabular}


Par conséquent, les valeurs propres pour $p=4$ sont de la forme :

$$
\left\{\begin{aligned}
\mathrm{VPR}_{1} & =-\frac{8}{L} \sum_{z \equiv 0 \bmod [4]}^{r}\left\{\Re\left(Z_{\mathrm{e}}^{z}\right)\left(\frac{\sin (z \pi \mathcal{R})}{z \pi}\right)^{2}\right\}-\frac{4 R_{\mathrm{ch}}}{L+4 L_{\mathrm{ch}}} \\
\mathrm{VPR}_{2} & =-\frac{8}{L} \sum_{z \equiv 2 \bmod [4]}^{r}\left\{\Re\left(Z_{\mathrm{e}}^{z}\right)\left(\frac{\sin (z \pi \mathcal{R})}{z \pi}\right)^{2}\right\} \\
\mathrm{VPC}_{1} & =-\frac{4}{L} \sum_{z \equiv 1 \bmod [4]}^{r}\left\{\left[\Re\left(Z_{\mathrm{e}}^{z}\right)-\jmath \Im\left(Z_{\mathrm{e}}^{z}\right)\right]\left(\frac{\sin (z \pi \mathcal{R})}{z \pi}\right)^{2}\right\} \\
\mathrm{VPC}_{2} & =\left(\mathrm{VPC}_{1}\right)^{*}
\end{aligned}\right.
$$

CAS GÉNÉRAL POUR $p$ QUELCONQUE. - Les résultats obtenus pour $p=3$ et $p=4$ (Éq. (B.3) et (B.6)) semblent répondre à des loıs particulières qui nous permettent de généraliser à un nombre quelconque de cellules

En effet, nous pouvons remarquer que la première valeur propre $V_{P R}$ est réelle et ne dépend que des harmoniques à $p$ fois la fréquence de découpage. Ainsi, nous pouvons écrire :

$$
\begin{aligned}
& \forall p \text { entier, } \\
& \mathrm{VPR}_{1}=-\frac{2 p}{L} \sum_{z=0 \bmod [p]}^{r}\left\{\Re\left(Z_{\mathrm{e}}^{z}\right)\left(\frac{\sin (z \pi \mathcal{R})}{z \pi}\right)^{2}\right\}-\frac{p R_{\mathrm{ch}}}{L+p \overline{L_{\mathrm{ch}}}} .
\end{aligned}
$$

En ce qui concerne les autres valeurs propres, nous pouvons remarquer que si le nombre de cellules est pair, il y a obligatoirement une autre valeur propre réelle notée $\mathrm{VPR}_{2}$. La généralisation de la relation obtenue pour $p=4$. nous permet d'écrire

$$
\begin{aligned}
& \forall p \text { pair, } \\
& \mathrm{VPR}_{2}=-\frac{2 p}{L} \sum_{z \equiv \frac{p}{2} \bmod [p]}^{r}\left\{\Re\left(Z_{\mathrm{e}}^{z}\right)\left(\frac{\sin (z \pi \mathcal{R})}{z \pi}\right)^{2}\right\} .
\end{aligned}
$$

Les autres valeurs propres étant complexes, il y en a $P$ telles que :

$$
\left\{\begin{array}{l}
P=p-1 \text { si } p \text { est impair } \\
P=p-2 \text { si } p \text { est pair. }
\end{array}\right.
$$

Nous les noterons $\mathrm{VPC}_{x}$ avec $x \in\{1, \ldots, P\}$. De plus, elles sont conjuguées deux à deux, nous pouvons donc généraliser en écrivant .

$$
\begin{aligned}
& \forall p \text { entier, } \forall k \in\left\{1, \ldots, \frac{P}{2}\right\}, \\
& \left\{\begin{aligned}
\mathrm{VPC}_{2 k-1}= & -\frac{p}{L} \sum_{z \equiv k \bmod [p]}^{r}\left\{\left[\Re\left(Z_{\mathrm{e}}^{z}\right)-\jmath \Im\left(Z_{\mathrm{e}}^{z}\right)\right]\left(\frac{\sin (z \pi \mathcal{R})}{z \pi}\right)^{2}\right\} \\
& -\frac{p}{L} \sum_{z \equiv(p-k) \bmod [p]}^{r}\left\{\left[\Re\left(Z_{\mathrm{e}}^{z}\right)+\jmath \Im\left(Z_{\mathrm{e}}^{z}\right)\right]\left(\frac{\sin (z \pi \mathcal{R})}{z \pi}\right)^{2}\right\} \\
\mathrm{VPC}_{2 k}= & \left(\mathrm{VPC}_{2 k-1}\right)^{*}
\end{aligned}\right.
\end{aligned}
$$

Les relations analytiques obtenues de manière empirique (Éq. (B.7, B.8, B.9)) pour un nombre quelconque de cellules ont été validées par comparaison de leurs résultats avec ceux obtenus par le modèle développé dans la première partie de l'article jusqu'à vingt cellules en parallèle 


\section{Annexe C}

\section{Influence du rapport cyclique sur les valeurs propres complexes}

Pour cette étude, nous prendrons comme hypothèse que le rééquilibrage n'est pas assuré car il y a au moins une valeur propre complexe à partie réelle nulle. D'après les relations obtenues dans l'équation 1 nous pouvons écrire que :

$$
\Re\left(\operatorname{VPC}_{2 k}\right)=\Re\left(\operatorname{VPC}_{2 k-1}\right)=-\frac{2 p}{L}\left\{\Re\left(Z_{\mathrm{e}}^{k}\right) \times \operatorname{Fonc}(k)+\Re\left(Z_{\mathrm{e}}^{p-k}\right) \times \operatorname{Fonc}(p-k)\right\} .
$$

En supposant que l'impédance d'entrée est correctement dimensionnée ( $. e . \Re\left(Z_{\mathrm{e}}^{k}\right)$ et $\Re\left(Z_{\mathrm{e}}^{p-k}\right)$ non nuls), nous avons donc :

$$
\left\{\begin{array}{lll}
\Re\left(Z_{\mathrm{e}}^{k}\right)>0 & \text { et } & \operatorname{Fonc}(k)=\left(\frac{\sin (k \pi \mathcal{R})}{k \pi}\right)^{2} \geq 0 \\
\Re\left(Z_{\mathrm{e}}^{p-k}\right)>0 & \text { et } & \operatorname{Fonc}(p-k)=\left(\frac{\sin ((p-k) \pi \mathcal{R})}{(p-k) \pi}\right)^{2} \geq 0
\end{array}\right.
$$

Ainsi, la partie réelle des valeurs propres complexes peut quand même être nulle si Fonc $(k)=$ Fonc $(k)=$ Fonc $(p-k)=0$. Or,

$$
\left\{\begin{array}{l}
\text { Fonc }(k)=0 \quad \Rightarrow \sin (k \pi \mathcal{R})=0 \quad \Rightarrow \exists \alpha \text { entier, } / \alpha=k \mathcal{R} \\
\operatorname{Fonc}(p-k)=0 \Rightarrow \sin ((p-k) \pi \mathcal{R})=0 \Rightarrow \exists \beta \text { entier, } / \beta=(p-k) \mathcal{R}
\end{array}\right.
$$

Par conséquent, Fonc $(k)=\operatorname{Fonc}(p-k)=0$ implique que :

$$
\exists \alpha, \beta \text { entiers, tel que } \frac{\alpha}{k}=\frac{\beta}{p-k} \text { donc } \alpha \times p=(\alpha+\beta) k
$$

D'après l'équation (C.4), on peut donc dire que $\alpha \times p$ est divisible par $k$ puisque $\alpha+\beta$ est un nombre entier. De plus, d'après la relation (C.3) et comme $\mathcal{R}<1$, on peut affirmer que $k$ ne divise pas $\alpha$ car $\alpha<k$, donc $k$ divise nécessairement $p$. Or $k \in\left\{1, \ldots, \frac{P}{2}\right\}$, ainsi nous pouvons déduire que $p$ n'est pas un nombre premier.

Nous avons ainsi montré que s'il n'y a pas de rééquilibrage possible pour une certaine valeur de rapport cyclique alors c'est que $p$ n'est pas premier. Par contraposition, nous pouvons donc dire que si $p$ est premier alors il y a rééquilibrage quelle que soit la valeur du rapport cyclique.

\section{Bibliographie}

[1] Davancens P. et Meynard T.A., Étude des convertisseurs multicellulaires parallèles : I. Modélisation, J. Phys. III France 7 (1997) 141-158. 